Proyecciones

Vol. 19, $\mathrm{N}^{\circ} 1$, pp. 65-94, May 2000

Universidad Católica del Norte

Antofagasta - Chile

\title{
FIXED POINTS PARAMETERS FOR TEICHMÜLLER SPACE OF CLOSED RIEMANN SURFACES
}

\author{
RUBÉN A. HIDALGO \\ Universidad Técnica Federico Santa María, Chile \\ and \\ GUSTAVO LABBE * \\ Universidad de La Serena, Chile
}

\begin{abstract}
In this note we provide a set of parameters for the Teichmüller space, of genus $g \geq 2$, given by fixed points of some special set of generators for the uniformizing Fuchsian groups. Explicit computations are given in low genus.
\end{abstract}

SUBJCLASS : Primary 30F40 30F60.

KEYWORDS : Kleinian groups, Teichmüller space

*Was partially supported by a project miniduc and DIULS $120-2-28$ and the first one by project USM 971223 and presidential chair of geometry 


\section{Introduction}

Given a finitely generated Fuchsian group $F$, acting on the hyperbolic plane $\mathbf{H}=\{z \in \mathbf{C}: \operatorname{Im}(z)>0\}$, we have associated its Teichmüller space (see next section). If we denote by $S$ the Riemann surface uniformized by $F$, that is, $S=\mathbf{H} / F$, then this space parametrizes all Riemann surfaces structures, modulo homotopy, that the surface $S$ may carry (preserving punctures and holes). It is well known that such a space is a real analytic simply-connected manifold of finite dimension [1] and that it can be identified to a component of a semi-algebraic set inside some $\mathbf{R}^{n}[3]$.

In the present note, we proceed to obtain a global set of coordinates for the Teichmüller space of a co-compact Fuchsian group of genus $g \geq 2$. These coordinates are given by fixed points of a special set of generators of the Fuchsian group and permit us to identify the Teichmüller space of genus $g \geq 2$ with an explicit semi-algebraic set inside $\mathbf{R}^{8 g-9}$ defined by $(2 g-3)$ polynomials.

The particularity of this set of fixed points parameters is that no multipliers or traces are used. Another explicit parameters (involving traces or multipliers) can be seen in [7], [6], [10], [8], [9], [11], [12], [15], [17] and [19], for instance.

The difficulty to read from the trace or multiplier the presence of parabolic elements makes sense to look for coordinates from which parabolic transformations can be readed. In fact, this will be the case if one wants to look boundary points in deformation spaces.

\section{Description of the parameter space $\mathcal{T}_{g}$}

To give the description of these parameters we need some notation. Set

- $L_{1}=\left(x_{1}, s_{1}, w_{1}\right) \in \mathbf{R}^{3}$ such that $x_{1}<0<1<s_{1}<w_{1}<u_{1}$;

- $L_{j}=\left(w_{j}, s_{j}, z_{j}, y_{j}, x_{j}, v_{j}\right) \in \mathbf{R}^{6}$ with $w_{j}<s_{j}<z_{j}<y_{j}<x_{j}<$ $v_{j}$, for each $j=2, \ldots, g-1$;

- $L_{g}=\left(v_{g}, x_{g}, y_{g}, z_{g}, s_{g}, w_{g}\right) \in \mathbf{R}^{6}$ with $v_{g}<x_{g}<y_{g}<z_{g}<s_{g}<$ $w_{g}$. 
Define the open set $\mathcal{K}_{g} \subset \mathbf{R}^{8 g-9}$ defined by the tupels

$$
\begin{gathered}
\left(L_{1}, L_{2}, q_{1}, L_{3}, q_{2}, L_{4}, \ldots, q_{g-3}, L_{g-1}, L_{g}, t_{g-3}, t_{g-4}, \ldots, t_{1}\right) \in \\
\mathbf{R}^{8 g-9} \text { so that } \\
w_{1}<w_{2}, \quad v_{j}<q_{j-1}<w_{j+1}, \quad \text { for } j=2, \ldots, g-2, \\
v_{g-1}<v_{g} \text { and } w_{g}<t_{g-3}<t_{g-4}<\cdots<t_{1} .
\end{gathered}
$$

Set the following real numbers:

$$
\begin{aligned}
u_{1}= & \frac{w_{1} x_{1}}{w_{1}+x_{1}-1} ; \\
u_{2}= & \frac{w_{2} x_{2}-y_{2} z_{2}}{w_{2}+x_{2}-y_{2}-z_{2}} ; \\
u_{j}= & \frac{v_{j} w_{j} x_{j}-w_{j} x_{j} y_{j}-w_{j} x_{j} z_{j}-v_{j} y_{j} z_{j}+w_{j} y_{j} z_{j}+x_{j} y_{j} z_{j}}{v_{j} w_{j}+v_{j} x_{j}-w_{j} x_{j}-v_{j} y_{j}-v_{j} z_{j}+y_{j} z_{j}}, \\
& \text { for } j=3, \ldots, g ; \\
r_{1}= & \frac{x_{1}\left(s_{1}-w_{1}\right)}{s_{1}\left(x_{1}-w_{1}+1\right)-x_{1}} ; \\
r_{j}= & \frac{-s_{j} w_{j} y_{j}+w_{j} x_{j} y_{j}+s_{j} x_{j} z_{j}-w_{j} x_{j} z_{j}+w_{j} y_{j} z_{j}-x_{j} y_{j} z_{j}}{s_{j}\left(x_{j}-w_{j}-y_{j}+z_{j}\right)+w_{j} y_{j}-x_{j} z_{j}}, \\
& \text { for } j=2, \ldots, g ;
\end{aligned}
$$

Consider the following rational expressiones :

$$
\begin{aligned}
& R_{1}=\frac{u_{1}\left(u_{1}-x_{1}\right)\left(u_{1}-u_{2}\right)\left(u_{1}-v_{2}\right)}{\left(u_{1}-w_{1}\right)\left(u_{1}-1\right)\left(u_{1}-t_{1}\right)\left(u_{1}-q_{1}\right)}-1, \text { for } g \geq 4 ; \\
& R_{1}=\frac{u_{1}\left(u_{1}-x_{1}\right)\left(u_{1}-u_{2}\right)\left(u_{1}-v_{2}\right)}{\left(u_{1}-w_{1}\right)\left(u_{1}-1\right)\left(u_{1}-u_{3}\right)\left(u_{1}-\left(u_{3}\right)\right)}-1, \text { for } g=3 ; \\
& R_{1}=\frac{u_{1}\left(u_{1}-x_{1}\right)\left(u_{1}-w_{2}\right)\left(u_{1}-z_{2}\right)}{\left(u_{1}-w_{1}\right)\left(u_{1}-1\right)\left(u_{1}-x_{2}\right)\left(u_{1}-y_{2}\right)}-1, \text { for } g=2 ; \\
& R_{2}=\frac{\left(t_{1}-u_{2}\right)\left(u_{1}-q_{1}\right)\left(t_{1}-v_{2}\right)}{\left(t_{1}-u_{1}\right)\left(u_{2}-q_{1}\right)\left(q_{1}-v_{2}\right)}-\frac{\left(q_{1}-t_{2}\right)\left(t_{1}-u_{3}\right)\left(t_{1}-v_{3}\right)\left(q_{1}-q_{2}\right)}{\left(t_{1}-t_{2}\right)\left(q_{1}-u_{3}\right)\left(q_{1}-v_{3}\right)\left(t_{1}-q_{2}\right)}, \\
& \quad \text { for } g \geq 4 ;
\end{aligned}
$$


$R_{2}=\frac{\left(u_{3}-u_{2}\right)\left(u_{1}-v_{3}\right)\left(u_{3}-v_{2}\right)}{\left(u_{3}-u_{1}\right)\left(u_{2}-v_{3}\right)\left(v_{3}-v_{2}\right)}-\frac{\left(v_{3}-w_{3}\right)\left(u_{3}-x_{3}\right)\left(u_{3}-y_{3}\right)\left(v_{3}-z_{3}\right)}{\left(u_{3}-w_{3}\right)\left(v_{3}-x_{3}\right)\left(u v-y_{3}\right)\left(u_{3}-z_{3}\right)}$

for $g=3$;

$R_{3}=\frac{\left(t_{1}-u_{2}\right)\left(u_{2}-q_{1}\right)\left(u_{1}-v_{2}\right)}{\left(u_{1}-u_{2}\right)\left(v_{2}-q_{1}\right)\left(t_{1}-v_{2}\right)}-\frac{\left(u_{1}-u_{2}\right)\left(v_{2}-q_{1}\right)\left(t_{1}-v_{2}\right)-e w}{\left(v_{2}-w_{2}\right)\left(v_{2}-x_{2}\right)\left(v_{2}-y_{2}\right)\left(u_{2}-z_{2}\right)}$

for $g \geq 3$;

$R_{4}=\frac{\left(u_{g-1}-u_{g}\right)\left(u_{g}-v_{g-1}\right)\left(t_{g-3}-v_{g}\right)\left(q_{g-3}-v_{g}\right)}{\left(t_{g-3}-u_{g}\right)\left(u_{g}-q_{g-3}\right)\left(u_{g-1}-v_{g}\right)\left(v_{g-1}-v_{g}\right)}$

$\frac{\left(v_{g}-w_{g}\right)\left(u_{g}-x_{g}\right)\left(u_{g}-y_{g}\right)\left(v_{g}-z_{g}\right)}{\left(u_{g}-w_{q}\right)\left(v_{g}-x_{g}\right)\left(v_{g}-y_{g}\right)\left(u_{g}-z_{g}\right)}, \quad$ for $g \geq 4$

$R_{3+j}=\frac{\left(v_{j+1}-w_{j+1}\right)\left(u_{j+1}-x_{j+1}\right)\left(u_{j+1}-y_{j+1}\right)\left(v_{j+1}-z_{j+1}\right)}{\left(u_{j+1}-w_{j+1}\right)\left(v_{j+1}-x_{j+1}\right)\left(v_{j+1}-y_{j+1}\right)\left(u_{j+1}-z_{j+1}\right)}-$

$\frac{\left(u_{j+1}-t_{j}\right)\left(t_{j-1}-v_{j+1}\right)\left(q_{j-1}-v_{j+1}\right)\left(u_{j+1}-q_{j}\right)}{\left(t_{j-1}-u_{j+1}\right)\left(u_{j+1}-q_{j-1}\right)\left(t_{j}-v_{j+1}\right)\left(v_{j+1}-q_{j}\right)}$,

for $j=2, \ldots, g-3$ and $g \geq 5$;

$$
\begin{aligned}
R_{g+1}= & \frac{\left(v_{g-1}-w_{g-1}\right)\left(u_{g-1}-x_{g-1}\right)\left(u_{g-1}-y_{g-1}\right)\left(v_{g-1}-z_{g-1}\right)}{\left(u_{g-1}-w_{g-1}\right)\left(v_{g-1}-x_{g-1}\right)\left(v_{g-1}-y_{g-1}\right)\left(u_{g-1}-z_{g-1}\right)}- \\
& \frac{\left(v_{g-1}-w_{g-1}\right)\left(u_{g-1}-x_{g-1}\right)\left(u_{g-1}-y_{g-1}\right)\left(v_{g-1}-z_{g-1}\right)}{\left(u_{g-1}-u_{g}\right)\left(t_{g-3}-v_{g-1}\right)\left(q_{g-3}-v_{g-1}\right)\left(u_{g-1}-v_{g}\right)},
\end{aligned}
$$

for $g \geq 4$; 


$$
\begin{aligned}
R_{g+j}= & \frac{\left(u_{j+1}-t_{j}\right)\left(t_{j}-v_{j+1}\right)\left(t_{j-1}-q_{j}\right)\left(q_{j-1}-q_{j}\right)}{\left(t_{j-1}-t_{j}\right)\left(t_{j}-q_{j-1}\right)\left(u_{j+1}-q_{j}\right)\left(v_{j+1}-q_{j}\right)}- \\
& \frac{\left(q_{j}-t_{j+1}\right)\left(t_{j}-u_{j+2}\right)\left(t_{j}-v_{j+2}\right)\left(q_{j}-q_{j+1}\right)}{\left(t_{j}-t_{j+1}\right)\left(q_{j}-u_{j+2}\right)\left(q_{j}-v_{j+2}\right)\left(t_{j}-q_{j+1}\right)}, \\
\text { for } j=2, \ldots, g-4 \text { and } g \geq 6 ; & \\
R_{2 g-3}= & \frac{\left(q_{g-3}-u_{g}\right)\left(t_{g-3}-u_{g-1}\right)\left(t_{g-3}-v_{g-1}\right)\left(q_{g-3}-v_{g}\right)}{\left(t_{g-3}-u_{g}\right)\left(q_{g-3}-u_{g-1}\right)\left(q_{g-3}-v_{g-1}\right)\left(t_{g-3}-v_{g}\right)}- \\
& \frac{\left(u_{g-2}-t_{g-3}\right)\left(t_{g-3}-v_{g-2}\right)\left(t_{g-4}-q_{g-3}\right)\left(q_{g-4}-q_{g-3}\right)}{\left(t_{g-4}-t_{g-3}\right)\left(t_{g-3}-q_{g-4}\right)\left(u_{g-2}-q_{g-3}\right)\left(v_{g-2}-q_{g-3}\right)},
\end{aligned}
$$

for $g \geq 5$.

Now we define the semi-algebraic set $\mathcal{T}_{g} \subset \mathbf{R}^{8 g-9}$ defined by the tupels in $\mathcal{K}_{g}$ satisfying $R_{1}=R_{2}=\cdots=R_{2 g-3}=0$ and

(i) $x_{1}<r_{1}<0$;

(ii) $w_{1}<u_{1}<u_{2}<w_{2}$;

(iii) $y_{j}<r_{j}<x_{j}$, for $j=2, \ldots, g-1$;

(iv) $x_{g}<r_{g}<y_{g}$;

(v) $v_{j}<q_{j-1}<u_{j+1}$, for $j=2, \ldots, g-2$;

(vi) $w_{g}<u_{g}<t_{g-3}$.

In section 6 we proceed to show the following result.

Theorem 1. The Teichmüller space of a Fuchsian group of genus $g \geq 2$ can be identified with the semi-algebraic set $\mathcal{T}_{g}$.

In genus two the above result can be made more explicit as follows (see section 7): 
Corollary 1. The Teichmüller space of genus 2 can be identified with the semi-algebraic set $\mathcal{T}_{2} \subset \mathbf{R}^{6}$ formed by the 6-tupels $\left(x_{1}, s_{1}, w_{1}, w_{2}, s_{2}, y_{2}\right)$ satisfying the following inequalities:

$x_{1}<0<1<s_{1}<w_{1}<1-x_{1} \quad$ and $\quad \frac{w_{1} x_{1}}{w_{1}+x_{1}-1}<w_{2}<s_{2}<z_{2}<y_{2}$,

where

$$
z_{2}=\frac{u_{1}\left(1-x_{1}+x_{2}\right)-x_{2}}{\left(u_{1}-x_{1}\right)}
$$

In section 8 we have also computed the above for the case of genus three Riemann surfaces.

We would like to thank the referee for her/his suggestions and in special for pointing us a mistake in the original statement of lemma 1.

\section{Teichmüller Space}

Let $F$ be a finitely generated Fuchsian group that uniformizes a Riemann surface $S=\mathbf{H} / F$. The surface $S$ is obtained from a closed Riemann surface of genus $g$ after deleting $l$ points and $k$ closed discs. We say that $S$ or $F$ has signature $(g, l, k)$.

The Teichmüller space of $F$ or $S$, denoted by $T(S)$ or $T(F)$, is defined as follows. Let us consider the collection $\operatorname{Hom}_{f}(F, P G L(2, \mathbf{C}))$ consisting of all faithful representations $\theta: F \rightarrow P G L(2, \mathbf{C})$ so that $\theta(F)$ is again a Fuchsian group of signature $(g, k, l)$.

We say that two representations $\theta_{1}, \theta_{2} \in \operatorname{Hom}_{f}(F, P G L(2, \mathbf{C}))$ are equivalent if there is a Möbius transformation $A$ so that $\theta_{2}(\gamma)=$ $A \theta_{1}(\gamma) A^{-1}$, for all $\gamma \in F$.

The set of equivalence classes is the Teichmüller space of $F$ (or $S$ ).

If we have that the group $F$ is non-elementary and $A, B \in F$ are so that Fix $(A), F i x(B)$ and Fix $(A B)$ are pairwise disjoint, then we have that each representation $\theta \in \operatorname{Hom}_{f}(F, P G L(2, \mathbf{C}))$ has a unique equivalent representation $\theta^{*}$ so that $a\left(\theta^{*}(A)\right)=0, a\left(\theta^{*}(A B)\right)=1$ and $a\left(\theta^{*}(B)\right)=\infty$ (see next section for the definition of these values). In particular, in this situation we can think of the Teichmüller space of $F$ as the set of normalized representations of $F$. 
Sources for the real and complex analytic theory of Teichmüller spaces are W. Abbikof's book [1] and Nag's book [18].

\section{Fixed Points of Möbius Transformations}

A conformal automorphism of the Riemann sphere is called a Möbius transformation. The general form of such a transformation is $B(z)=$ $\frac{a z+b}{c z+d}$, where $a, b, c, d$ are complex numbers so that $a d-b c \neq 0$. There is a natural isomorphism between the group of Möbius transformations and the projective linear group $P G L(2, \mathbf{C})$ given by

$$
B(z)=\frac{a z+b}{c z+d} \mapsto\left[\begin{array}{ll}
a & b \\
c & d
\end{array}\right] .
$$

A Möbius transformation has at least one and at most two fixed points. If the transformation has exactly one fixed point, then it is called parabolic. In the other case, if it is conjugated to a rotation, then we call it an elliptic transformation; otherwise, it is a loxodromic transformation. If $B$ is a loxodromic transformation and $p \in \mathbf{C}$ a fixed point, then we call it attracting or repelling if $\left\|B^{\prime}(p)\right\|<1$ or $\left\|B^{\prime}(p)\right\|>1$, respectively. If one of the fixed points is infinity, then it is called attracting or repelling if the other fixed point is repelling or attracting, respectively. A loxodromic element has exactly one repelling fixed point and one attracting fixed point.

If $B$ is a Möbius transformation different from the identity so that $B^{2} \neq I$, then we can define complex values $a(B)$ and $r(B)$ as follows. (i) If $B$ is loxodromic, then $a(B)$ and $r(B)$ are the attracting and repelling fixed points of $B$, respectively. (ii) If $B$ is parabolic, then $a(B)=r(B)$ is its unique fixed point. (iii) If $B$ is elliptic, then $a(B)$ and $r(B)$ are the fixed points of $B$ for which there exists a Möbius transformation $H$ so that $H B H^{-1}(z)=\lambda z(\|\lambda\|=1, \operatorname{Im}(\lambda)>0)$, $H(a(B))=\infty$ and $H(r(B))=0$. We denote the set of fixed points of the transformation $B$ by $F(B)$.

The following two results are the basic facts for the construction of fixed point parameters of Teichmüller spaces. 
Lemma 1. Let $A, B$ in $P G L(2, \mathbf{C})$ be so that $A^{2} \neq I, B^{2} \neq I$, $(A B)^{2} \neq I$ and $F(A) \cap F(B)=\emptyset$. Then the pair $(A, B)$ is uniquely determined by the 6-tupel $(r(A), r(B), r(A B), a(A), a(B), a(A B))$.

Lemma 2. Let $A, B$ and $C$ be transformations in $P G L(2, \mathbf{C})$ so that neither of $A, B, C, A B$ has order two, and they satisfy $C A C^{-1}=A B$. If any of two these transformations have a common fixed point, then the triple $(A, B, C)$ is uniquely determined by the 6-tupel $(a(A), a(B)$, $a(C), a(A B), r(A), r(A B))$.

\section{Fixed Points Parameters For Fuchsian Groups of Signature $(0,0,3)$}

Let us consider a Fuchsian group $F$ that uniformizes a triple holed sphere $S$.

It is known that $F$ has a set of generators $A$ and $B$ so that the axe of the transformations $A, B$ and $A B$ have a configuration, up to isometries of the hyperbolic plane, as shown in figure 1 . In that case, we have that the fixed points satisfy the following inequalities

$$
r(A)=x<a(A)=0<a(A B)=1<r(A B)=w<r(B)=u .
$$

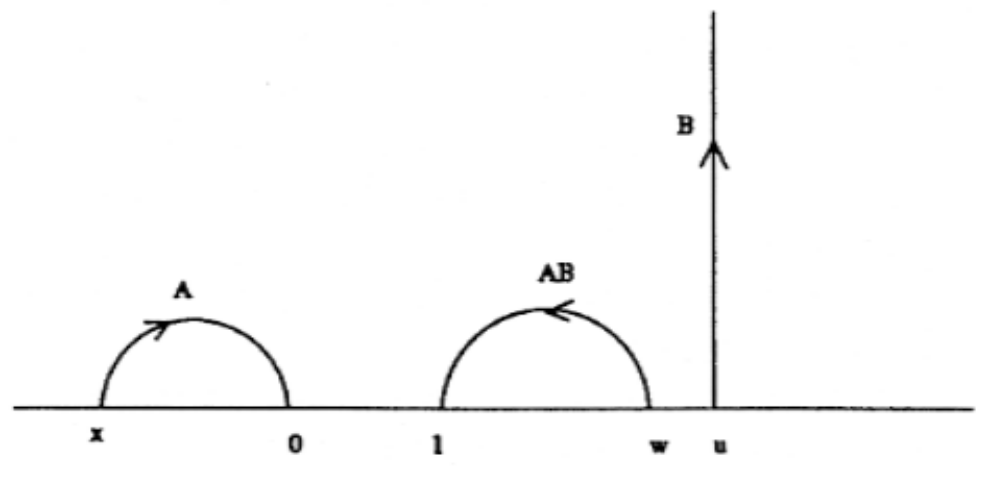

Figure 1 
As consequence of the proof of lemma 1, the multipliers of the transformations $A, B$ and $A B$ are given by:

$$
\begin{aligned}
k_{A} & =\frac{u(x-w)(x-1)}{w(u-x)}, \\
k_{B} & =\frac{u(u-x)}{(u-w)(u-1)}, \\
k_{A B} & =\frac{w(x-w)(u-1)}{(u-w)(x-1)} .
\end{aligned}
$$

We obtain that the Teichmüller space of triple holed spheres can be embedded into the open set

$$
V=\left\{(x, w, u) \in \mathbf{R}^{3}: x<0<1<w<u\right\}
$$

On the other hand, if we are given a tripe $(x, w, u) \in V$, then we may construct three simple geodesics $L_{1}, L_{2}$ and $L_{3}$ inside the hyperbolic plane, so that $L_{1}$ connects $x$ with $0, L_{2}$ connects 1 with $w$ and $L_{3}$ connects $u$ with $\infty$.

Construct the geodesic lines $M_{1,2}, M_{1,3}$ and $M_{2,3}$, so that $M_{i, j}$ is orthogonal to $L_{i}$ and $L_{j}$. Let $\sigma_{i, j}$ be the reflection along $M_{i, j}$.

Set $A=\sigma_{1,2} \sigma_{1,3}$ and $B=\sigma_{1,3} \sigma_{2,3}$. Then we have that $a(A)=0$, $a(B)=\infty, a(A B)=1, r(A)=x, r(B)=u$ and $r(A B)=w$. Moreover, the group generated by $A$ and $B$ is of signature $(0,0,3)$. It follows that the triple $(x, w, u)$ is obtained from a normalized representation of a Fuchsian group $F$ of signature $(0,0,3)$. As a consequence of the above, we get that the Teichmüller space of signature $(0,0,3)$ is naturally identified with the set $V$.

Remark. In the boundary of $V$ we have the following components: $V_{1}=\{(0, w, u): 1<w<u\}, V_{2}=\{(x, 1, u): x<0,1<u\}$, $V_{3}=\{(x, w, \infty): x<0<1<w\}, V_{1,2}=\{(0,1, u): 1<u\}$, $V_{1,3}=\{(0, w, \infty): 1<w\}, V_{2,3}=\{(x, 1, \infty): x<0\}$ and $V_{1,2,3}=$ $\{(0,1, \infty)\}$. We have that $V_{1}, V_{2}$ and $V_{3}$ give us models for the Teichmüller space of a sphere with two holes and one puncture; $V_{1,2}, V_{1,3}$ and $V_{2,3}$ give us models for the Teichmüller space of a sphere with two 
punctures and one hole; and $V_{1,2,3}$ is model for the Teichmüller space of a sphere with three punctures.

\section{Fixed Points Parameters For Fuchsian Groups of Signature $(1,0,1)$}

Assume we have a Fuchsian group $F$ that uniformizes a genus one Riemann surface with a hole. It is well known that there is a set of generators $A, B$ and $C$ so that $C A C^{-1}=A B$. The axe of the transformations $A, B, C$ and $A B$ have a configuration as shown in figure 2 (up to isometries of the hyperbolic plane). In this case, we have $a(A)=0, a(A B)=1, a(B)=\infty$ and

$r(A)=x<r(C)=r<0<1<a(C)=s<r(A B)=w<r(B)=u$.

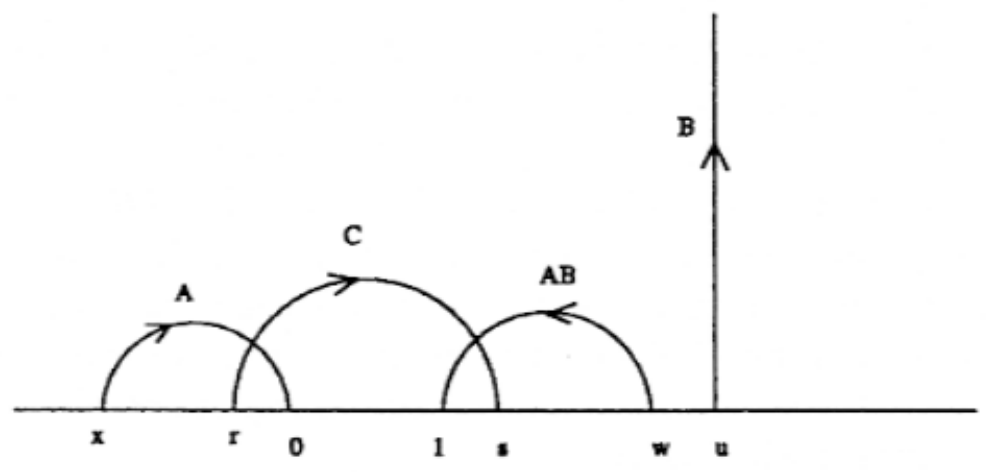

Figure 2

As consequence of lemma 2, we have that the transformations $A$, $B$ and $C$ are uniquely determined as follows: 


$$
\begin{aligned}
k_{C} & =\frac{s(s-x)(w-1)}{x(s-w)(s-1)}, \\
r(C) & =r=\frac{x(s-w)}{s(x-w+1)-x} .
\end{aligned}
$$

The relation $C A C^{-1}=A B$ asserts that $k_{A}=k_{A B}$. This is equivalent to the equality

$$
u=\frac{w x}{w+x-1} .
$$

Inequality $w<u$ is equivalent to $w<1-x$. Inequality $r<0$ is equivalent to $x<\frac{-s}{s-1}(1-w)$, which is true since $\frac{-s}{s-1}(1-w)>0$. Inequality $x<r$ holds since $x<w$ asserts that $s(x-w+1)<x$.

In this way, we have that the Teichmüller space of a genus one surface with a hole can be embedded inside the open set

$$
W=\left\{(x, s, w) \in \mathbf{R}^{3}: x<0<1<s<w<1-x\right\} .
$$

On the other hand, if we are given a triple $(x, s, w) \in W$, we may construct the values $r$ and $u$ as above. We proceed to construct the geodesic lines $L_{1}, L_{2}$ and $L_{3}$ in the hyperbolic plane so that $L_{1}$ connects $x$ with $0, L_{2}$ connects 1 and $w, L_{3}$ connects $u$ with $\infty$. We construct the geodesic lines $M_{i, j}$ orthogonal to the lines $L_{i}$ and $L_{j}$ $(i<j)$.

If $\sigma_{i, j}$ is the reflection on the line $M_{i, j}$, then we may construct the transformations $A=\sigma_{1,2} \sigma_{1,3}$ and $B=\sigma_{1,3} \sigma_{2,3}$. In this way, $a(A)=0$, $a(A B)=1, a(B)=\infty, r(A)=x, r(A B)=w$ and $r(B)=u$.

The relations on the coordinates $x, s$ and $w$ asserts that $k_{A}=k_{A B}$.

Consider the transformation $C$ determined by the conditions that $a(C)=s, r(C)=r$ and $k_{C}=\frac{s(s-x)(w-1)}{x(s-w)(s-1)}$. We have then that $C(x)=w$ and $C(0)=1$. It follows that $C A C^{-1}$ has attracting and repelling fixed points 1 and $w$, respectively. Since we have that $k_{A}=k_{A B}$, we have that $C A C^{-1}=A B$.

As a consequence, the triple $(x, s, w)$ is determined by a normalized represention of a Fuchsian group $F$ of signature $(1,0,1)$. In particular, the Teichmüller space of signature $(1,0,1)$ is naturally identified to the open set $W$. 
Remark. In the boundary of $W$ we have the following component $W_{1}=\{(x, s, 1-x): x<0<1<s<1-x\}$ (corresponding to $w=x-1$, that is, the transformation $B$ is a parabolic). We have that $W_{1}$ gives us a model for the Teichmüller space of a genus one Riemann surface with a puncture.

\section{Fixed Points Parameters For Fuchsian Groups of Signature $(g, 0,0)$}

Now we can combine the two sections above to obtain a model, by fixed points, of the Teichmüller space of a surface of genus $g \geq 2$.

Let us consider a Fuchsian group $F$ of genus $g \geq 2$ acting on the hyperbolic plane $\mathbf{H}$. We may find a set of generators $A_{1}, B_{1}, C_{1}, A_{2}$, $B_{2}, C_{2}, \ldots, A_{g}, B_{g}, C_{g}, A_{g+1}, B_{g+1}, A_{g+2}, B_{g+2}, \ldots, A_{2 g-2}$ and $B_{2 g-2}$, so that

(1) $C_{i} A_{i} C_{i}^{-1}=A_{i} B_{i}$, for $i=1, \ldots, g$;

(2) $B_{1}=B_{g+1}\left(\right.$ for $g=2$ this means $\left.B_{1}=B_{2}\right)$;

(3) $A_{g+1}=B_{g+2}\left(\right.$ for $g \geq 3$; for $g=3$ this means $\left.A_{4}=B_{3}\right)$;

(4) $A_{g+1} B_{g+1}=B_{2}($ for $g \geq 3)$;

(5) $A_{2 g-2} B_{2 g-2}=B_{g}($ for $g \geq 4)$;

(6) $A_{g+j}=B_{j+1}, j=2, \ldots, g-2($ for $g \geq 4)$;

(7) $A_{g+j} B_{g+j}=B_{g+j+1}, j=2, \ldots, g-3$ (for $g \geq 5$ ).

Set $x_{i}=r\left(A_{i}\right), y_{i}=a\left(A_{i}\right), z_{i}=a\left(A_{i} B_{i}\right), w_{i}=r\left(A_{i} B_{i}\right), u_{i}=r\left(B_{i}\right)$, $v_{i}=a\left(B_{i}\right), r_{i}=r\left(C_{i}\right)$ and $s_{i}=a\left(C_{i}\right)$, for $i=1, \ldots, g$.

Up to conjugation, we may assume that $a\left(A_{1}\right)=0, a\left(B_{1}\right)=\infty$ and $a\left(A_{1} B_{1}\right)=1$. In this way, the other fixed points must satisfy the following inequalities (see figure 3 ): 


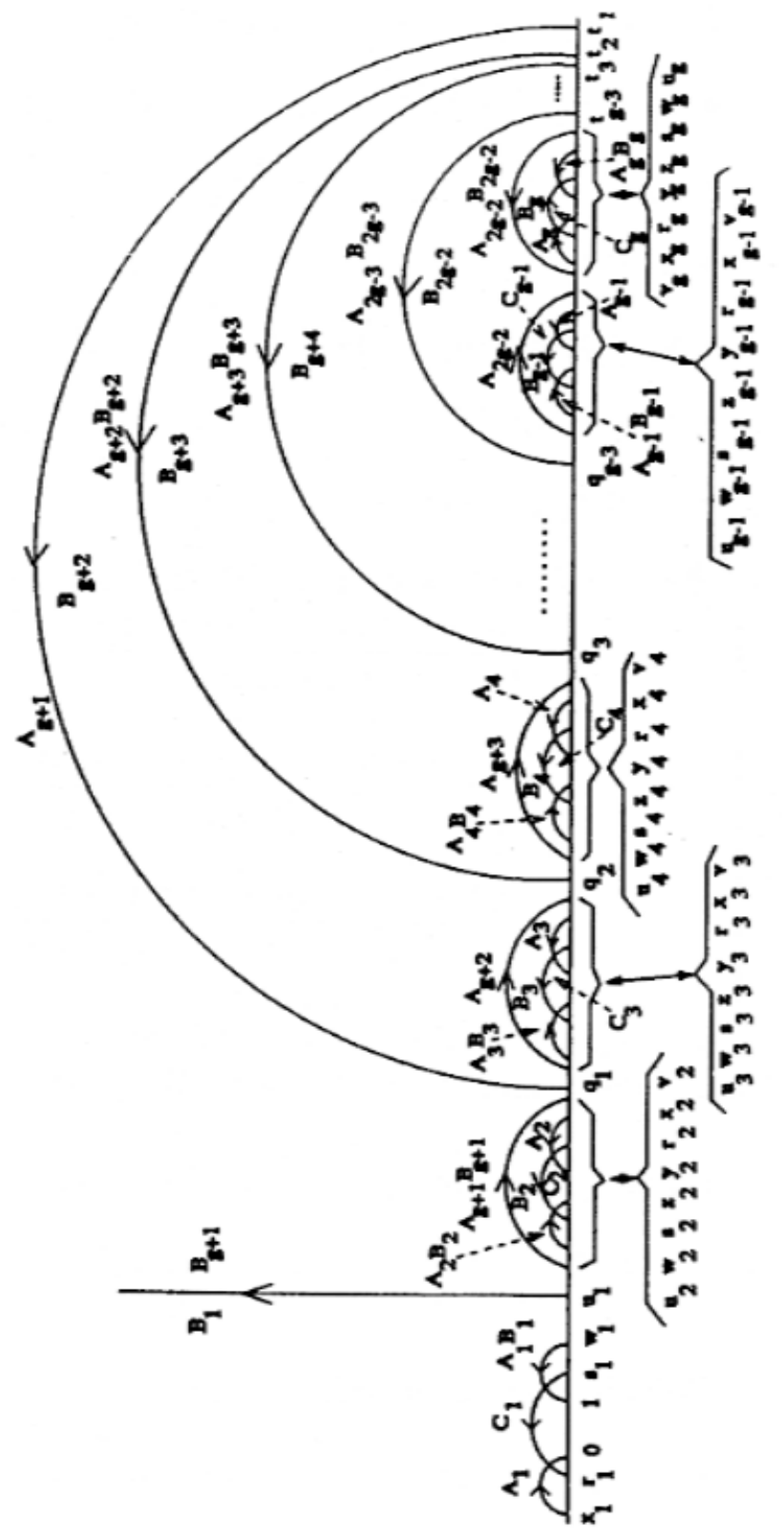

Figure 3 
- $x_{1}<r_{1}<y_{1}=0<z_{1}=1<s_{1}<w_{1}<u_{1}$;

- $u_{j}<w_{j}<s_{j}<z_{j}<y_{j}<r_{j}<x_{j}<v_{j}$, for each $j=2, \ldots, g-1$;

- $v_{g}<x_{g}<r_{g}<y_{g}<z_{g}<s_{g}<w_{g}<u_{g}$;

- $u_{1}<u_{2}$;

- $v_{j}<q_{j-1}<u_{j+1}$, for $j=2, \ldots, g-2$;

- $v_{g-1}<v_{g}$

- $u_{g}<t_{g-3}<t_{g-4}<\cdots<t_{1}$.

Relations in (1) give us the following for $i=1, \ldots, g$ :

$(*)\left\{\begin{aligned} k_{C_{i}} & =\frac{\left(s_{i}-x_{i}\right)\left(s_{i}-y_{i}\right)\left(w_{i}-z_{i}\right)}{\left(s_{i}-w_{i}\right)\left(x_{i}-y_{i}\right)\left(s_{i}-z_{i}\right)} \\ r\left(C_{i}\right) & =r_{i}=\frac{-s_{i} w_{i} y_{i}+w_{i} x_{i} y_{i}+s_{i} x_{i} z_{i}-w_{i} x_{i} z_{i}+w_{i} y_{i} z_{i}-x_{i} y_{i} z_{i}}{s_{i}\left(x_{i}-w_{i}-y_{i}+z_{i}\right)+w_{i} y_{i}-x_{i} z_{i}} \\ u_{i} & =\frac{v_{i} w_{i} x_{i}-w_{i} x_{i} y_{i}-w_{i} x_{i} z_{i}-v_{i} y_{i} z_{i}+w_{i} y_{i} z_{i}+x_{i} y_{i} z_{i}}{v_{i} w_{i}+v_{i} x_{i}-w_{i} x_{i}-v_{i} y_{i}-v_{i} z_{i}+y_{i} z_{i}} .\end{aligned}\right.$

The equalities (2)-(7) are equivalent (in our situation) to the equalities of the respective multipliers. We proceed to write down them.

Equality (2), for $g \geq 4$, is equivalent to:

$u_{1}\left(u_{1}-x_{1}\right)\left(u_{1}-u_{2}\right)\left(u_{1}-v_{2}\right)-\left(u_{1}-w_{1}\right)\left(u_{1}-1\right)\left(u_{1}-t_{1}\right)\left(u_{1}-q_{1}\right)=0$.

Equality (2), for $g=3$, is equivalent to:

$u_{1}\left(u_{1}-x_{1}\right)\left(u_{1}-u_{2}\right)\left(u_{1}-v_{2}\right)-\left(u_{1}-w_{1}\right)\left(u_{1}-1\right)\left(u_{1}-u_{3}\right)\left(u_{1}-v_{3}\right)=0$.

Equality (2), for $g=2$, is equivalent to:

$u_{1}\left(u_{1}-x_{1}\right)\left(u_{1}-w_{2}\right)\left(u_{1}-z_{2}\right)-\left(u_{1}-w_{1}\right)\left(u_{1}-1\right)\left(u_{1}-x_{2}\right)\left(u_{1}-y_{2}\right)=0$. 
Equality (3), for $g \geq 4$, is equivalent to:

$\frac{\left(t_{1}-u_{2}\right)\left(u_{1}-q_{1}\right)\left(t_{1}-v_{2}\right)}{\left(t_{1}-u_{1}\right)\left(u_{2}-q_{1}\right)\left(q_{1}-v_{2}\right)}-\frac{\left(q_{1}-t_{2}\right)\left(t_{1}-u_{3}\right)\left(t_{1}-v_{3}\right)\left(q_{1}-q_{2}\right)}{\left(t_{1}-t_{2}\right)\left(q_{1}-u_{3}\right)\left(q_{1}-v_{3}\right)\left(t_{1}-q_{2}\right)}=0$.

Equality (3), for $g=3$, is equivalent to:

$\frac{\left(u_{3}-u_{2}\right)\left(u_{1}-v_{3}\right)\left(u_{3}-v_{2}\right)}{\left(u_{3}-u_{1}\right)\left(u_{2}-v_{3}\right)\left(v_{3}-v_{2}\right)}-\frac{\left(v_{3}-w_{3}\right)\left(u_{3}-x_{3}\right)\left(u_{3}-y_{3}\right)\left(v_{3}-z_{3}\right)}{\left(u_{3}-w_{3}\right)\left(v_{3}-x_{3}\right)\left(v_{3}-y_{3}\right)\left(u_{3}-z_{3}\right)}=0$.

Equality (4) is equivalent to:

$\frac{\left(t_{1}-u_{2}\right)\left(u_{2}-q_{1}\right)\left(u_{1}-v_{2}\right)}{\left(u_{1}-u_{2}\right)\left(v_{2}-q_{1}\right)\left(t_{1}-v_{2}\right)}-\frac{\left(v_{2}-w_{2}\right)\left(u_{2}-x_{2}\right)\left(u_{2}-y_{2}\right)\left(v_{2}-z_{2}\right)}{\left(u_{2}-w_{2}\right)\left(v_{2}-x_{2}\right)\left(v_{2}-y_{2}\right)\left(u_{2}-z_{2}\right)}=0$.

Equality (5) is equivalent to:

$$
\begin{gathered}
\frac{\left(u_{g-1}-u_{g}\right)\left(u_{g}-v_{g-1}\right)\left(t_{g-3}-v_{g}\right)\left(q_{g-3}-v_{g}\right)}{\left(t_{g-3}-u_{g}\right)\left(u_{g}-q_{g-3}\right)\left(u_{g-1}-v_{g}\right)\left(v_{g-1}-v_{g}\right)}- \\
\quad \frac{\left(v_{g}-w_{g}\right)\left(u_{g}-x_{g}\right)\left(u_{g}-y_{g}\right)\left(v_{g}-z_{g}\right)}{\left(u_{g}-w_{g}\right)\left(v_{g}-x_{g}\right)\left(v_{g}-y_{g}\right)\left(u_{g}-z_{g}\right)}=0 .
\end{gathered}
$$

Equality (6), for $j=2, \ldots, g-3$, is equivalent to:

$$
\begin{aligned}
& \frac{\left(v_{j+1}-w_{j+1}\right)\left(u_{j+1}-x_{j+1}\right)\left(u_{j+1}-y_{j+1}\right)\left(v_{j+1}-z_{j+1}\right)}{\left(u_{j+1}-w_{j+1}\right)\left(v_{j+1}-x_{j+1}\right)\left(v_{j+1}-y_{j+1}\right)\left(u_{j+1}-z_{j+1}\right)}- \\
& \quad \frac{\left(u_{j+1}-t_{j}\right)\left(t_{j-1}-v_{j+1}\right)\left(q_{j-1}-v_{j+1}\right)\left(u_{j+1}-q_{j}\right)}{\left(t_{j-1}-u_{j+1}\right)\left(u_{j+1}-q_{j-1}\right)\left(t_{j}-v_{j+1}\right)\left(v_{j+1}-q_{j}\right)}=0 .
\end{aligned}
$$

Equality (6), for $j=g-2$ is equivalent to:

$$
\frac{\left(v_{g-1}-w_{g-1}\right)\left(u_{g-1}-x_{g-1}\right)\left(u_{g-1}-y_{g-1}\right)\left(v_{g-1}-z_{g-1}\right)}{\left(u_{g-1}-w_{g-1}\right)\left(v_{g-1}-x_{g-1}\right)\left(v_{g-1}-y_{g-1}\right)\left(u_{g-1}-z_{g-1}\right)}-
$$




$$
\frac{\left(u_{g-1}-u_{g}\right)\left(t_{g-3}-v_{g-1}\right)\left(q_{g-3}-v_{g-1}\right)\left(u_{g-1}-v_{g}\right)}{\left(t_{g-3}-u_{g-1}\right)\left(u_{g-1}-q_{g-3}\right)\left(u_{g}-v_{g-1}\right)\left(v_{g-1}-v_{g}\right)}=0 .
$$

Equality (7), for $j=2, \ldots, g-4$, is equivalent to:

$$
\begin{gathered}
\frac{\left(u_{j+1}-t_{j}\right)\left(t_{j}-v_{j+1}\right)\left(t_{j-1}-q_{j}\right)\left(q_{j-1}-q_{j}\right)}{\left(t_{j-1}-t_{j}\right)\left(t_{j}-q_{j-1}\right)\left(u_{j+1}-q_{j}\right)\left(v_{j+1}-q_{j}\right)}- \\
\frac{\left(q_{j}-t_{j+1}\right)\left(t_{j}-u_{j+2}\right)\left(t_{j}-v_{j+2}\right)\left(q_{j}-q_{j+1}\right)}{\left(t_{j}-t_{j+1}\right)\left(q_{j}-u_{j+2}\right)\left(q_{j}-v_{j+2}\right)\left(t_{j}-q_{j+1}\right)}=0 .
\end{gathered}
$$

Equality (7), for $j=g-3$, is equivalent to:

$$
\begin{gathered}
\frac{\left(q_{g-3}-u_{g}\right)\left(t_{g-3}-u_{g-1}\right)\left(t_{g-3}-v_{g-1}\right)\left(q_{g-3}-v_{g}\right)}{\left(t_{g-3}-u_{g}\right)\left(q_{g-3}-u_{g-1}\right)\left(q_{g-3}-v_{g-1}\right)\left(t_{g-3}-v_{g}\right)}- \\
\frac{\left(u_{g-2}-t_{g-3}\right)\left(t_{g-3}-v_{g-2}\right)\left(t_{g-4}-q_{g-3}\right)\left(q_{g-4}-q_{g-3}\right)}{\left(t_{g-4}-t_{g-3}\right)\left(t_{g-3}-q_{g-4}\right)\left(u_{g-2}-q_{g-3}\right)\left(v_{g-2}-q_{g-3}\right)}=0 .
\end{gathered}
$$

In this way, we obtain $(8 g-9)$ fixed points that determine the others, and are related by the $(2 g-3)$ equations comming from relations (2)-(7). This gives us the $(6 g-6)$ real parameters as corresponds for describing the Teichmüller space of $F$. As a consequence of this, we have that the Teichmüller space of signature $(g, 0,0)$ is embedded in the open set $\mathcal{T}_{g}$ (as defined in the first section).

To obtain that every tupel in $\mathcal{T}_{g}$ is determined by some normalized representation of a Fuchsian group of signature $(g, 0,0)$, we use the constructions used above together the Klein-Maskit combination theorems [16]. To be more precise; for each tupel $\left(L_{1}, \ldots, L_{g}\right)$ in $\mathcal{T}_{g}$ (as defined in the first section), we proceed to construct the the values $u_{j}$, $r_{j}, k_{C_{j}}$ given by the formulae in $(*)$, for all $j=1, \ldots, g$.

Now we construct the transformations $A_{j}, B_{j}$ and $C_{j}$ so that $a\left(A_{j}\right)=y_{j}, r\left(A_{j}\right)=x_{j}, a\left(B_{j}\right)=v_{j}, r\left(B_{j}\right)=u_{j}, a\left(A_{j} B_{j}\right)=z_{j}$, $r\left(A_{j} B_{j}\right)=w_{j}, a\left(C_{j}\right)=s_{j}, r\left(C_{j}\right)=r_{j}$ and the multiplier of $C_{j}$ is $k_{C_{j}}$.

Each pair $\left(A_{j}, B_{j}\right)$ produces a group of signature $(0,0,3)$. The equations we have, from (2) to (7) above, permit us to use the firts 
Klein-Maskit's combination theorem for gluing all the above groups to produce a group of signature $(0,0,2 g)$.

The values of $r_{j}$ and $k_{C_{j}}$ make possible to use the second KleinMaskit's combination theorem to produce a group of signature $(g, 0,0)$ as desired.

\section{Genus Two}

In this section we consider the information obtained in the last section for the particular case of genus two. We follow the same notation as last section.

Let us consider a Fuchsian group uniformizing a genus two surface. It has generators $A_{1}, B_{1}, C_{1}, A_{2}, B_{2}, C_{2}$, and relations $C_{1} A_{1} C_{1}^{-1}=$ $A_{1} B_{1}, C_{2} A_{2} C_{2}^{-1}=A_{2} B_{2}$ and $B_{1}=B_{2}$. We assume as before that $a\left(A_{1}\right)=0, a\left(B_{1}\right)=\infty$ and $a\left(A_{1} B_{1}\right)=1$.

In this case, we have that (see figure 4$)$ :

$x_{1}<r_{1}<0<1<s_{1}<w_{1}<u_{1}<w_{2}<s_{2}<z_{2}<y_{2}<r_{2}<x_{2}$.

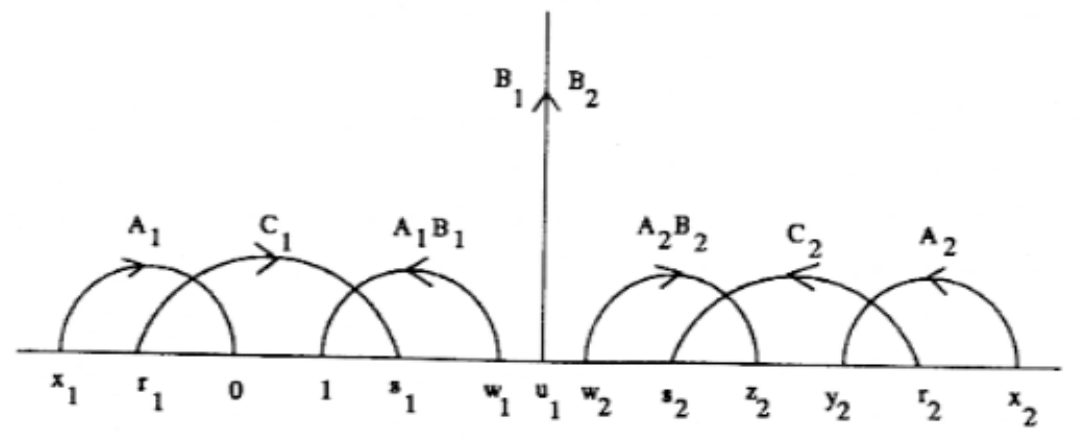

Figure 4 
The relation $C_{1} A_{1} C_{1}^{-1}=A_{1} B_{1}$ asserts that:

$$
\begin{aligned}
& u_{1}=\frac{w_{1} x_{1}}{\left(w_{1}+x_{1}-1\right)}, \\
& r_{1}=\frac{x_{1}\left(s_{1}-w_{1}\right)}{s_{1}\left(x_{1}-w_{1}+1\right)-x_{1}} .
\end{aligned}
$$

The relation $C_{2} A_{2} C_{2}^{-1}=A_{2} B_{2}$, together the above value of $u_{1}$, asserts:

$$
\begin{aligned}
x_{2} & =\frac{u_{1}\left(-w_{2}+y_{2}+z_{2}\right)-y_{2} z_{2}}{\left(u_{1}-w_{2}\right)} \\
r_{2} & =\frac{-s_{2} w_{2} y_{2}+w_{2} x_{2} y_{2}+s_{2} x_{2} z_{2}-w_{2} x_{2} z_{2}+w_{2} y_{2} z_{2}-x_{2} y_{2} z_{2}}{s_{2}\left(x_{2}-w_{2}-y_{2}+z_{2}\right)+w_{2} y_{2}-x_{2} z_{2}} .
\end{aligned}
$$

Equality $B_{1}=B_{2}$ is equivalent, in our situation, to $k_{B_{1}}=k_{B_{2}}$. This gives us two possibilities for $z_{2}$ :

$$
z_{2} \in\left\{\frac{2 u_{1}^{2}-\left(1+x_{1}+x_{2}\right) u_{1}+x_{2}}{\left(u_{1}-x_{1}\right)}, \frac{\left(1-x_{1}+x_{2}\right) u_{1}-x_{2}}{\left(u_{1}-x_{1}\right)}\right\} .
$$

The fact that $x_{2} \neq u_{1}$ asserts that the above two possible values for $z_{2}$ can never coincide. Since the Teichmüller space is connected, we have that only one of these values is the correct one. To see which one is the real value for $z_{2}$, we consider a particular case. This case is the symmetric situation: $w_{2}=2 u_{1}-w_{1}, s_{2}=2 u_{1}-s_{1}, z_{2}=2 u_{1}-1$, $y_{2}=2 u_{1}, r_{2}=2 u_{1}-r_{1}$ and $x_{2}=2 u_{1}-x_{1}$. This Fuchsian group of genus two uniformizes a Riemann surface of genus two with a symmetry (induced by reflection on the axis of $B_{1}$ ).

In this way, we have in general that

$$
z_{2}=\frac{\left(1-x_{1}+x_{2}\right) u_{1}-x_{2}}{\left(u_{1}-x_{1}\right)}
$$

where the value of $u_{1}$ is given above.

As a consequence of all above, we have that a model for the Teichmüller space of genus two is given by the semi algebraic set $\mathcal{T}_{2} \subset \mathbf{R}^{6}$ 
formed by the 6 -tupels $\left(x_{1}, s_{1}, w_{1}, y_{2}, s_{2}, w_{2}\right)$ satisfying the following inequalities:

$$
\begin{gathered}
x_{1}<0<1<s_{1}<w_{1}<1-x_{1} \\
\frac{w_{1} x_{1}}{w_{1}+x_{1}-1}<w_{2}<s_{2}<z_{2}<y_{2}
\end{gathered}
$$

where $z_{2}$ is as before.

\section{Genus Three}

Let us consider the particular case of genus three Riemann surfaces. A genus three Fuchsian group is generated by transformations $A_{1}, B_{1}$, $C_{1}, A_{2}, B_{2}, C_{2}, A_{3}, B_{3}, C_{3}, A_{4}, B_{4}$, so that:

(i) $C_{j} A_{j} C_{j}^{-1}=A_{j} B_{j}$, for $j=1,2,3$;

(ii) $B_{1}=B_{4}$;

(iii) $B_{2}=A_{4} B_{4}$;

(iv) $B_{3}=A_{4}$.

Up to conjugation, $a\left(A_{1}\right)=0, a\left(A_{1} B_{1}\right)=1$ and $a\left(B_{1}\right)=\infty$. The fixed points of the above transformations satisfy (see figure 5 ):

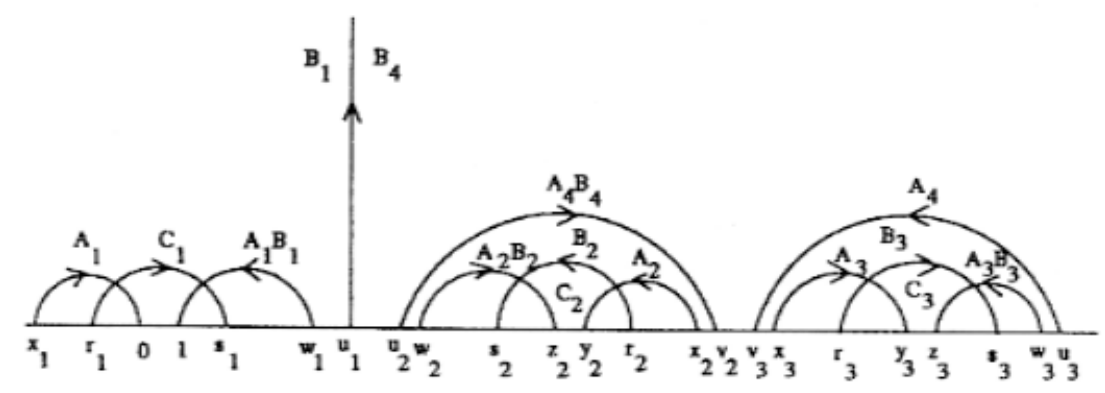

Figure 5 


$$
\begin{gathered}
x_{1}<r_{1}<0<1<s_{1}<w_{1}<u_{1}, \\
u_{1}<u_{2}<w_{2}<s_{2}<z_{2}<y_{2}<r_{2}<x_{2}<v_{2}, \\
v_{2}<v_{3}<x_{3}<r_{3}<y_{3}<z_{3}<s_{3}<w_{3}<u_{3} .
\end{gathered}
$$

Equalities in (i) gives us the values:

$$
\begin{aligned}
r_{1} & =\frac{x_{1}\left(s_{1}-w_{1}\right)}{s_{1}\left(1+x_{1}-w_{1}\right)-x_{1}}, \\
r_{2}= & \frac{w_{2} z_{2} y_{2}-w_{2} z_{2} x_{2}+w_{2} y_{2} x_{2}-z_{2} y_{2} x_{2}-w_{2} y_{2} s_{2}+z_{2} x_{2} s_{2}}{w_{2} y_{2}-z_{2} x_{2}+s_{2}\left(-w_{2}+z_{2}-y_{2}+x_{2}\right)}, \\
r_{3}= & \frac{x_{3} y_{3} z_{3}-x_{3} y_{3} w_{3}+x_{3} z_{3} w_{3}-y_{3} z_{3} w_{3}-x_{3} z_{3} s_{3}+y_{3} w_{3} s_{3}}{x_{3} z_{3}-y_{3} w_{3}-x_{3} s_{3}+y_{3} s_{3}-z_{3} s_{3}+w_{3} s_{3}}, \\
u_{1}= & \frac{x_{1} w_{1}}{x_{1}+w_{1}-1}, \\
u_{2}= & \frac{w_{2} z_{2} y_{2}-w_{2} z_{2} x_{2}-w_{2} y_{2} x_{2}+z_{2} y_{2} x_{2}-z_{2} y_{2} v_{2}+w_{2} x_{2} v_{2}}{z_{2} y_{2}-w_{2} x_{2}+w_{2} v_{2}-z_{2} v_{2}-y_{2} v_{2}+x_{2} v_{2}} \\
u_{3}= & \frac{-v_{3} y_{3} z_{3}+x_{3} y_{3} z_{3}+v_{3} x_{3} w_{3}-x_{3} y_{3} w_{3}-x_{3} z_{3} w_{3}+y_{3} z_{3} w_{3}}{v_{3} x_{3}-v_{3} y_{3}-v_{3} z_{3}+y_{3} z_{3}+v_{3} w_{3}-x_{3} w_{3}} .
\end{aligned}
$$

Equality $B_{1}=B_{4}$ is equivalent to:

$$
\frac{u_{1}\left(u_{1}-x_{1}\right)\left(u_{1}-u_{2}\right)\left(u_{1}-v_{2}\right)}{\left(u_{1}-1\right)\left(u_{1}-w_{1}\right)\left(u_{1}-u_{3}\right)\left(u_{1}-v_{3}\right)}=1 \text {. }
$$

Equality $B_{2}=A_{4} B_{4}$ is equivalent to:

$$
\frac{\left(u_{2}-u_{3}\right)\left(u_{2}-v_{3}\right)\left(u_{1}-v_{2}\right)\left(u_{2}-w_{2}\right)\left(v_{2}-x_{2}\right)\left(v_{2}-y_{2}\right)\left(u_{2}-z_{2}\right)}{\left(u_{1}-u_{2}\right)\left(u_{3}-v_{2}\right)\left(v_{3}-v_{2}\right)\left(v_{2}-w_{3}\right)\left(u_{2}-x_{2}\right)\left(u_{2}-y_{2}\right)\left(v_{2}-z_{2}\right)}=1
$$

Equality $A_{4}=B_{3}$ is equivalent to: 


$$
\frac{\left(u_{2}-u_{3}\right)\left(u_{1}-v_{3}\right)\left(u_{3}-v_{2}\right)\left(u_{3}-w_{3}\right)\left(v_{3}-x_{3}\right)\left(v_{3}-y_{2}\right)\left(u_{3}-z_{3}\right)}{\left(u_{1}-u_{3}\right)\left(u_{2}-v_{3}\right)\left(v_{3}-v_{2}\right)\left(v_{3}-w_{3}\right)\left(u_{3}-x_{3}\right)\left(u_{3}-y_{3}\right)\left(v_{3}-z_{3}\right)}=1 .
$$

As consequence of the above, we have that the set $\mathcal{T}_{3}$ is defined by the 15-tupels:

$$
\left(x_{1}, s_{1}, w_{1}, w_{2}, s_{2}, z_{2}, y_{2}, x_{2}, v_{2}, v_{3}, x_{3}, y_{3}, z_{3}, s_{3}, w_{3}\right) \in \mathbf{R}^{15}
$$

so that

$$
x_{1}<0,
$$

$1<s_{1}<w_{1}<w_{2}<s_{2}<z_{2}<y_{2}<x_{2}<v_{2}<v_{3}<x_{3}<y_{3}<z_{3}<s_{3}$

$<w_{3}$, satisfying the extra inequalities:

$$
\begin{gathered}
x_{1}<r_{1}<0, \\
w_{1}<u_{1}<u_{2}<w_{2}, \\
y_{2}<r_{2}<x_{2}, \\
x_{3}<r_{3}<y_{3}, \\
w_{3}<u_{3},
\end{gathered}
$$

and satisfying the above rational equations.

\section{Proof of Lemmata 1 and 2}

\subsection{Proof of Lemma 1}

Let us consider a pair of Möbius transformations $(A, B)$ satisfying the hypotheses of our Theorem. We can normalize the pair so that $a(B)=\infty, a(A)=0$ and $a(A B)=1$. We must prove that the values $x=r(B), y=r(A)$ and $z=r(A B)$ determine uniquely the normalized pair $(A, B)$.

(I) $A$ and $B$ are not parabolic. The transformations $A$ and $B$ can be represented by the matrices: 


$$
\begin{aligned}
& B=\left(\begin{array}{cc}
k_{1}^{2} & x\left(1-k_{1}^{2}\right) \\
0 & 1
\end{array}\right), \\
& A=\left(\begin{array}{cc}
y & 0 \\
1-k_{2}^{2} & y k_{2}^{2}
\end{array}\right),
\end{aligned}
$$

where either $\left\|k_{j}^{2}\right\|>1$ or $k_{j}^{2}=e^{2 \pi i \theta_{j}}, \theta_{j} \in(0,1 / 2)$, for $j=1,2$.

We only need to prove that the multipliers $k_{1}^{2}$ and $k_{2}^{2}$ are uniquely determined by the values $x, y$ and $z$.

In this case, the transformation $A B$ has the following matrix representation:

$$
A B=\left(\begin{array}{cc}
y k_{1}^{2} & x y\left(1-k_{1}^{2}\right) \\
k_{1}^{2}\left(1-k_{2}^{2}\right) & x\left(1-k_{1}^{2}\right)\left(1-k_{2}^{2}\right)+y k_{2}^{2}
\end{array}\right) .
$$

Since 1 is a fixed point of $A B$, we have the equation:

$$
k_{2}^{2}\left(k_{1}^{2}+x\left(1-k_{1}^{2}\right)-y\right)=(1-y)\left(k_{1}^{2}+x\left(1-k_{1}^{2}\right)\right)
$$

The facts $y \neq 1, y \neq 0$ and $k_{2} \neq 0$ imply that $k_{1}^{2}+x\left(1-k_{1}^{2}\right)-y \neq 0$. In particular,

$$
k_{2}^{2}=\frac{(1-y)\left(k_{1}^{2}+x\left(1-k_{1}^{2}\right)\right)}{k_{1}^{2}+x\left(1-k_{1}^{2}\right)-y} .
$$

The fixed points of $A B$ are the roots of the quadratic polynomial in $w$ :

$$
k_{1}^{2}\left(1-k_{2}^{2}\right) w^{2}+\left[x\left(1-k_{1}^{2}\right)\left(1-k_{2}^{2}\right)+y k_{2}^{2}-y k_{1}^{2}\right] w-x y\left(1-k_{1}^{2}\right)=0 .
$$

In particular, $z=\frac{-x y\left(1-k_{1}^{2}\right)}{k_{1}^{2}\left(1-k_{2}^{2}\right)}$ and (since $\left.z \neq 0\right)$ 


$$
(* *) \quad k_{2}^{2}=\frac{x y\left(1-k_{1}^{2}\right)+z k_{1}^{2}}{k_{1}^{2} z} .
$$

The equality of the RHS of $(*)$ and $(* *)$ gives us the following equation to be satisfied by $k_{1}^{2}$ :

$$
k_{1}^{4}-k_{1}^{2}\left(\frac{x z(y-1)+x y(1-x)+(x-y)(z-x y)}{y(1-x)(z-x)}\right)+\frac{x(x-y)}{(1-x)(z-x)}=0 .
$$

The solutions to this equations are by $1,-1, k_{1}$ and $-k_{1}$, so we obtain:

$$
\begin{aligned}
k_{1}^{2} & =\frac{x(x-y)}{(1-x)(z-x)}, \\
k_{2}^{2} & =\frac{x(y-1)(y-z)}{z(x-y)} .
\end{aligned}
$$

(II) $B$ is parabolic and $A$ is not parabolic. In this case $A$ and $B$ have the following representation:

$$
\begin{aligned}
& B=\left(\begin{array}{ll}
1 & a \\
0 & 1
\end{array}\right), \\
& A=\left(\begin{array}{cc}
y & 0 \\
1-k_{2}^{2} & y k_{2}^{2}
\end{array}\right),
\end{aligned}
$$

where either $\left\|k_{2}^{2}\right\|>1$ or $k_{2}^{2}=e^{2 \pi i \theta}, \theta \in(0,1 / 2)$ and $a \neq 0$.

We need to see that $a$ and $k_{2}^{2}$ are uniquely determined by the values $y$ and $z$.

In this case, $A B$ has the following matrix representation:

$$
A B=\left(\begin{array}{cc}
y & a y \\
1-k_{2}^{2} & a\left(1-k_{2}^{2}\right)+y k_{2}^{2}
\end{array}\right) .
$$

The fact that 1 is a fixed point of $A B$ gives us the equation: 


$$
(1+a)(y-1)=k_{2}^{2}(y-1-a) .
$$

Since $k_{2} \neq 0$ and $y \neq 1$, we must have that $1+a=0$ if and only if $y=1+a$; in which case $y=0$, a contradiction. In particular, we get

$$
\text { (*) } \quad k_{2}^{2}=\frac{(1+a)(y-1)}{y-1-a} .
$$

The fixed points of the transformation $A B$ are the roots of the quadratic equation in $w$ :

$$
w^{2}+(a-y) w-\frac{a y}{1-k_{2}^{2}}=0 .
$$

It follows that $z=\frac{-a y}{1-k_{2}^{2}}$, and

$$
(* *) \quad k_{2}^{2}=\frac{z+a y}{z} .
$$

The equality of the RHS of $(*)$ and $(* *)$ implies the following equation to be satisfied by $a$ :

$$
a^{2} y+a y(z-y+1)=0
$$

Since $a \neq 0$ and $y \neq 0$, we obtain:

$$
\begin{gathered}
a=y-z-1, \\
k_{2}^{2}=\frac{(y-1)(y-z)}{z} .
\end{gathered}
$$

(III) $B$ is not parabolic and $A$ is parabolic. In this case, $A$ and $B$ have the following matrix representation:

$$
\begin{aligned}
& A=\left(\begin{array}{ll}
1 & 0 \\
b & 1
\end{array}\right), \\
& B=\left(\begin{array}{cc}
k_{1}^{2} & x\left(1-k_{1}^{2}\right) \\
0 & 1
\end{array}\right),
\end{aligned}
$$


where either $\left\|k_{1}^{2}\right\|>1$ or $k_{1}^{2}=e^{2 \pi i \theta}, \theta \in(0,1 / 2)$ and $b \neq 0$.

We need to see that $b$ and $k_{1}^{2}$ are uniquely determined by the values $x$ and $z$.

The transformation $A B$ has the following matrix representation:

$$
A B=\left(\begin{array}{cc}
k_{1}^{2} & x\left(1-k_{1}^{2}\right) \\
b k_{1}^{2} & b x\left(1-k_{1}^{2}\right)+1
\end{array}\right) .
$$

The fact that 1 is a fixed point of $A B$ implies the following equation:

$$
1+x(b-1)=k_{1}^{2}(1+x(b-1)-b) .
$$

Since $k_{1} \neq 0$, we must have that $1+x(b-1)=0$ if and only if $1+x(b-1)-b=0$, in which case $b=0$ a contradiction. In particular, we get

$$
\text { (*) } \quad k_{1}^{2}=\frac{1+x(b-1)}{1+x(b-1)-b} .
$$

The fixed points of the transformation $A B$ are the roots of the quadratic equation in $w$ :

$$
b k_{1}^{2} w^{2}+(b x+1)\left(1-k_{1}^{2}\right) w-x\left(1-k_{1}^{2}\right)=0 .
$$

It follows that $z=\frac{-x\left(1-k_{1}^{2}\right)}{b k_{1}^{2}}$, and

$$
(* *) \quad k_{1}^{2}=\frac{x}{x-z b} \text {. }
$$

The equality of the RHS of $(*)$ and $(* *)$ gives the following equation to be satisfied by $b$ :

$$
z x b^{2}+b(z(1-x)-x)=0 \text {. }
$$


Since $b \neq 0, x \neq 0$ and $z \neq 0$, we obtain:

$$
\begin{aligned}
b & =\frac{x+z(x-1)}{x z}, \\
k_{1}^{2} & =\frac{x^{2}}{(x-1)(x-z)} .
\end{aligned}
$$

(IV) $A$ and $B$ are parabolic. In this case, $A$ and $B$ have the following matrix representation:

$$
\begin{aligned}
& A=\left(\begin{array}{ll}
1 & 0 \\
b & 1
\end{array}\right), \\
& B=\left(\begin{array}{ll}
1 & a \\
0 & 1
\end{array}\right),
\end{aligned}
$$

where $a b \neq 0$. We need to see that $a$ and $b$ are uniquely determined by the value $z$. The transformation $A B$ has the following matrix representation:

$$
A B=\left(\begin{array}{cc}
1 & a \\
b & 1+a b
\end{array}\right) .
$$

Since 1 is a fixed point of $A B$, we have the following equation:

$$
a=b(1+a)
$$

The fact that $a \neq 0$ implies $a \neq-1$, and we obtain the equation:

$$
\text { (*) } \quad b=\frac{a}{1+a} .
$$

The fixed points of the transformation $A B$ are the roots of the quadratic equation in $w$ : 


$$
w^{2}+a w-\frac{a}{b}=0
$$

It follows that $z=-\frac{a}{b}$, and

$$
(* *) \quad b=-\frac{a}{z} .
$$

The equality of the RHS of $(*)$ and $(* *)$ implies:

$$
a=-1-z, \quad \text { and } \quad b=\frac{1+z}{z} .
$$

Remark. In the proof of lemma 1 we didn't use the fact that 1 is attracting fixed point of the transformation $A B$. We only used that it was just a fixed point of it. By considering this condition, we will have some inequalities to be satisfied by $x, y$ and $z$. Moreover, by writing out the inequalities satisfied by the multipliers $k_{1}$ and $k_{2}$ will give us some extra inequalities on them. All these inequalities will give the reciprocal of lemma 1, that is, to describe the set of parameters for which it is possible to reconstruct (uniquely by the above) the transformations $A$ and $B$. Since this is not necessary for the work in this note, we skip to write such a reciprocal.

\subsection{Proof of Lemma 2}

Relation $C A C^{-1}=A B$ is equivalent to the following equalities:

(i) $k_{A}=k_{A B}$;

(ii) $C(r(A))=r(A B)$;

(iii) $C(a(A))=a(A B)$.

Equalities (ii) and (iii) imply the values:

$$
\begin{aligned}
k_{C} & =\frac{(s-x)(s-y)(w-z)}{(s-w)(x-y)(s-z)}, \\
r(C) & =\frac{-s w y+w x y+s x z-w x z+w y z-x y z}{s(x-w-y+z)+w y-x z},
\end{aligned}
$$


where $x=r(A), y=a(A), z=a(A B), w=r(A B), u=r(B)$, $v=a(B)$ and $s=a(C)$.

Now, the transformation $C$ is uniquely determined by $r(C), a(C)$ and $k_{C}$. The transformations $A$ and $B$ are also uniquely determined by lemma 1 . Equality (i) is equivalent to:

$u v w+u v x-u w x-v w x-u v y+w x y-u v z+w x z+u y z+v y z-w y z-x y z=0$.

This last equation gives us the value of $u$ in function of the others.

\section{References}

[1] W. Abbikof. The real analytic theory of Teichmüller space. Lecture Notes in Mathematics 820, Springer, (1980).

[2] L. Bers. "On moduli of Riemann surfaces". (Mimeographed lecture notes), Eidgenossische Technische Hochschule, Zurig, (1964).

[3] H. Helling. "Diskrete Untergruppen von $S L_{2}(\mathbf{R})$ ". Inventiones Math. 17, pp. 217-299, (1972).

[4] R.A. Hidalgo. "The noded Schottky space". Proc. London Math. Soc. 73, pp. 385-403, (1996).

[5] R.A. Hidalgo. "Fixed point parameters for Fuchsian groups of type $(1,2,0)$ ". Accepted for publication in Revista Scientia.

[6] C. L. Earle. "Some intrinsic coordinates on Teichmüller space". Proc. Amer. Math. Soc. 83, pp. 527-531, (1981).

[7] C. L. Earle and A. Marden. "Geometric complex coordinates for Teichmüller space" (to appear)

[8] I. Kra. "Horocyclic coordinates for Riemann surfaces and moduli spaces. I: Teichmüller and Riemann spaces of Kleinian groups". J. of the Amer. Math. Soc. 3, pp. 499-578, (1990). 
[9] I. Kra, "Non-variational global coordinates for Teichmüller spaces", in Holomorphic Functions and Moduli II, Math. Sci. Res. Inst. Publ., vol. 11, Springer, Berlin, Tokyo, pp. 221-249, (1988).

[10] I. Kra and B. Maskit. "The deformation space of a Kleinian group". Amer. J. Math. 103, pp. 1065-1102, (1980).

[11] A. Marden. "Geometric complex coordinates for Teichmüller space". In Mathematical Aspects of String Theory (S. T. Yau, ed.), World Scientific, Singapore, pp. 341-364, (1987).

[12] B. Maskit. "Moduli of marked Riemann surfaces". Bull. Amer. Math. Soc. 80, pp. 773-777, (1974).

[13] B. Maskit. "Parameters for Fuchsian groups I: Signature $(0,4)$ ". Holomorphic Functions and Moduli II, pp. 251-265. Math. Sci. Res. Inst. Pub. 11. Springer - Verlag, New York, (1988).

[14] B. Maskit. "Parameters for Fuchsian groups II: topological type $(1,1)$ ". Annal. Acad. Sci. Fenn. Ser. A.I. 14, pp. 265-275, (1990).

[15] B. Maskit. "Explicit Matrices for Fuchsian groups". Preprint IHES/M/92/6 Février (1992).

[16] B. Maskit. Kleinian Groups. Grundlehren der Mathematischen Wissenschaften, vol. 287,Springer - Verlag, Berlin, Heildelberg, New York, (1988).

[17] C. Min. "New parameters of Teichmüller spaces of Riemann surfaces of genus two". Ann. Acad. Scie. Fenn., Series A.I. Mathematica, Vol.17, (1992).

[18] S. Nag. The complex analytic theory of Teichmüller spaces. Canadian Mathematical Society Series of Monographs and Advances texts. AWiley Interscience Pub. (1988).

[19] M. Seppala and T. Sorvali. "On geometric parametrization of Teichmüller spaces". Ann. Acad. Sci. Fenn. Series A. I. Mathematica, vol. 10, pp. 515-526, (1985). 
Received by : June 22, 1999.

Rubén A. Hidalgo

Departamento de Matemáticas

Universidad Técnica Federico Santa María

Valparaíso

Chile

and

Gustavo Labbé

Departamento de Matemáticas

Universidad de La Serena

La Serena

Chile 